\title{
Modification of Electric Transport Properties of CVD Graphene by Electrochemical Deposition of Cobalt Nanoparticles
}

\author{
V. Bayev*, J. Fedotova and U. Humennik \\ Institute for Nuclear Problems, Belarusian State University \\ Bobruiskaya Str. 11, 220030 Minsk, Belarus \\ *bayev@hep.by \\ S. Vorobyova and A. Konakow \\ Research Institute for Physical Chemical Problems \\ Belarusian State University \\ Leningradskaya Str. 9, 220030 Minsk, Belarus \\ A. Fedotov and I. Svito \\ Energy Physics Department \\ Faculty of Physics, Belarusian State University \\ Bobruyskaya Str. 5, 220030 Minsk, Belarus \\ M. Rybin and E. Obraztsova \\ A. M. Prokhorov General Physics Institute \\ Vavilov Str. 38, 119991 Moscow, Russia
}

Received 22 December 2018

Accepted 14 January 2019

Published 13 May 2019

\begin{abstract}
Electrochemical deposition of cobalt nanoparticles was used to modify carrier transport properties of single-layered CVD graphene at the $\mathrm{SiO}_{2}$-on-Si substrate. The structure of graphene with cobalt nanoparticles was analyzed by Raman spectroscopy and scanning electron microscopy. The effect of the deposited cobalt nanoparticles on the sheet resistance of graphene was studied in the temperature range of $4-300 \mathrm{~K}$.
\end{abstract}

Keywords: CVD graphene; electrochemical deposition; Co nanoparticles; sheet resistance.

\section{Introduction}

Graphene is supposed to be the most promising perspective material for creation of flexible transparent conductive films in optoelectronic devices. The main problem of this application of graphene is that the common method of synthesis, chemical vapor deposition (CVD) provides films with extremely high sheet resistance (ordinarily more than $500 \Omega / \square$ ) because of their polycrystalline structure. There are several methods to increase the electrical conductivity of CVD graphene such as doping, combining with metal nanowires and creating complex multilayer graphene-based structures. ${ }^{1,2}$ It was demonstrated that in the doped 
CVD graphene the sheet resistance could be lowered down to $100 \Omega / \square$. $^{2}$ At the same time, a reasonable sheet resistance is considered to be $\sim 50 \Omega / \square$.

\section{Experimental Methods}

In this paper, the modification of charge carrier transport properties was performed by electrochemical deposition of cobalt nanoparticles (Co NPs). Sheets of single-layered CVD graphene with the size of $10 \times 10 \mathrm{~mm}$ were fabricated by homemade cold-wall chemical vapor deposition on $25 \mu \mathrm{m}$ thick copper foil (99.8\% purity) from the methane precursor with nitrogen as a carrier gas. The fabrication of graphene and its characterization were described in detail elsewhere. ${ }^{3}$

The graphene sheets were transferred onto $\mathrm{SiO}_{2}$-on-Si substrate using the standard procedure of Polymethylmethacrylate (PMMA) spin-coating and further dissolving of $\mathrm{Cu}$ substrate in the ammonium persulfate solution. PMMA/graphene membrane is scooped onto the $\mathrm{SiO}_{2}$-on-Si substrate. Then PMMA layer was removed by acetone.

Electrochemical deposition of cobalt onto the surface of graphene on the $\mathrm{SiO}_{2}$-on-Si substrate was carried out at a constant current at room temperature. ${ }^{4}$ The electrolyte contained $1.0 \mathrm{~g} / \mathrm{L}$ of $\mathrm{CoSO}_{4} \cdot 7 \mathrm{H}_{2} \mathrm{O}$ and $0.064 \mathrm{~g} / \mathrm{L}$ of $\mathrm{NaCl}$ dissolved in distilled water. All reagents used in this experiment were of analytical grade and were used without further purification. The $\mathrm{SiO}_{2}$-on-Si substrate covered with a graphene layer was used as a cathode and with stainless steel plates as an anode and the electrode separation of about $5 \mathrm{~mm}$. The current density was $2.5 \mathrm{~mA} / \mathrm{cm}^{2}$ and the electrodeposition time was $30 \mathrm{~s}$. After the deposition, the samples obtained were thoroughly washed in distilled water and dried at room temperature and then analyzed.

\section{Results}

Raman spectra of the virgin and Co NPs modified graphene recorded with the scanning laser $(532 \mathrm{~nm})$ Raman microscope Confotec NR500 (Sol instruments) are represented in Fig. 1.

Raman spectroscopy does not reveal any significant changes in the graphene structure after the electrochemical deposition of Co NPs. The observed small shifts of G-peak $\left(\Delta_{G}=-3 \mathrm{~cm}^{-1}\right)$ and 2Dpeak $\left(\Delta_{2 D}=+2 \mathrm{~cm}^{-1}\right)$ could be caused by such factors as strains, contaminations, doping, etc. ${ }^{5,6}$

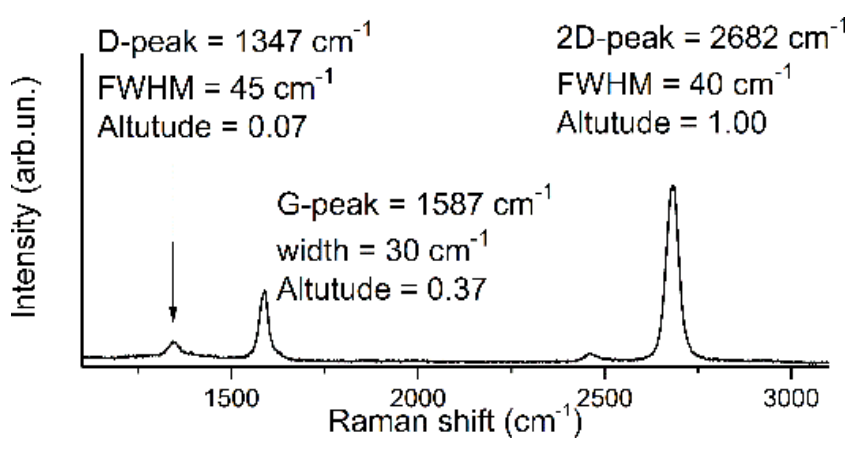

(a)

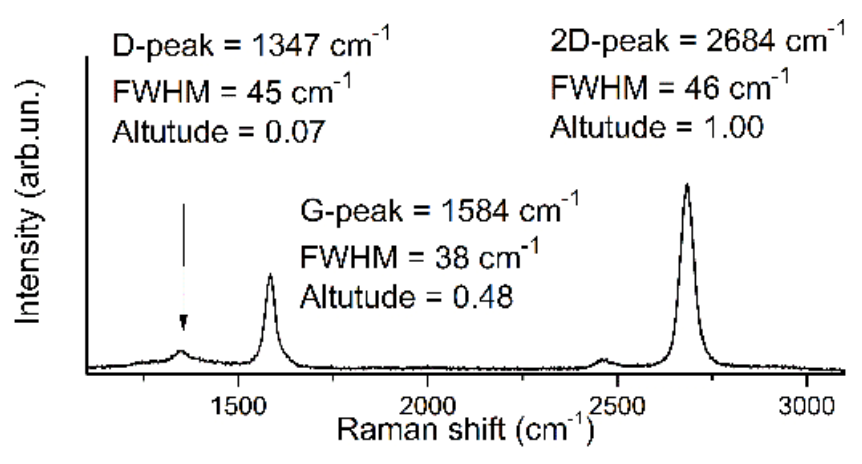

(b)

Fig. 1. Raman spectra of the virgin (a) and Co NPs modified graphene on the $\mathrm{SiO}_{2}$-on-Si substrate.

The results of scanning electron microscopy (SEM) obtained with a LEO-1455 microscope equipped with the energy dispersive X-ray spectrometer RONTEG are represented in Fig. 2. According to these images, Co NPs with sizes of about $200 \mathrm{~nm}$ and in the form of dendrites with sizes $\sim 1 \mu \mathrm{m}$ were deposited. Estimation of the area

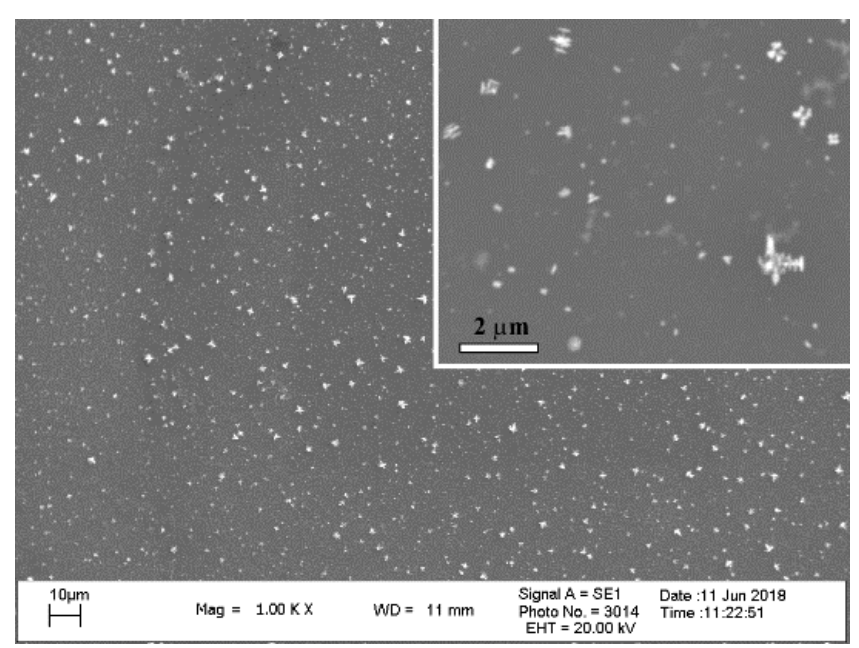

Fig. 2. SEM image of the Co NPs-modified graphene on the $\mathrm{SiO}_{2}$-on-Si substrate. 


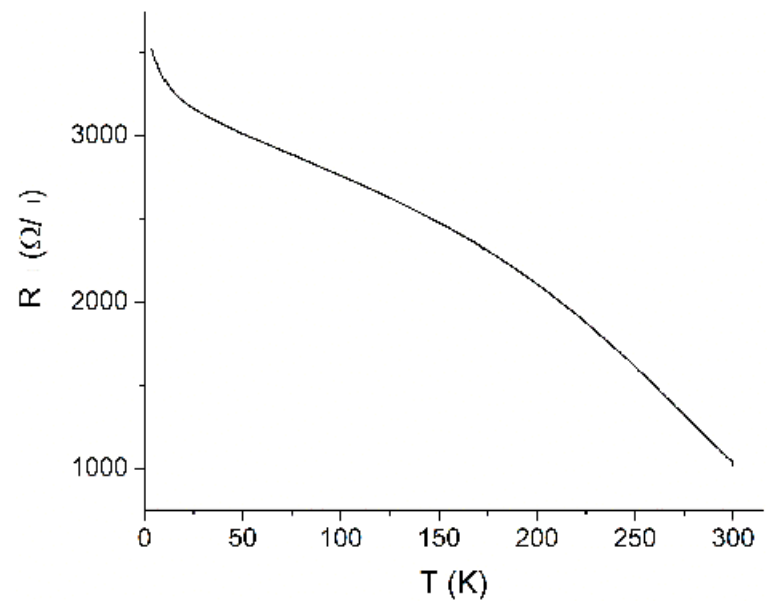

Fig. 3. Temperature dependence of sheet resistance of the virgin graphene on $\mathrm{SiO}_{2}$-on-Si substrate.

occupied by nanoparticles and dendrites gives about $5 \%$ of the whole sample area.

The four-probe electric resistance as a function of temperature, $R(T)$, of virgin and modified with Co NPs graphene was measured in a Cryogenics Ltd closed-cycle refrigerator system in the temperature range $2 K<T<300 \mathrm{~K}$. The measurements were performed on the samples mounted on the removable contact pad of the probe using 4 indium electric contacts with soldered thin copper rods. Temperature was stabilized with the precision down to $0.01 \mathrm{~K}$ using a LakeShore 331 controller. The error in the resistance measurements was mainly limited by the size of electric contacts and intercontact distances and was equal or less than $5 \%$. The resistance was represented in sheet resistance units $(\Omega / \square)$ calculated as $R_{\square}=R \cdot(W / L)$, where $W$ is the width of the current conductive channel (the graphene layer) and $L$ is the distance between the potential contacts.

The $R_{\square}(T)$ dependence of the virgin graphene at the $\mathrm{SiO}_{2}$-on-Si substrate is presented in Fig. 3. The sheet resistance at room temperature appears to be of $1000 \Omega / \square$ that is typical for CVD graphene. The resistance of graphene increases with the temperature decrease evidencing electric conductivity of the activation type (semiconducting). Such behavior can be assigned to the polycrystalline structure where graphene crystallites with low resistance are separated by high-defect grain boundaries. The resistance of the virgin graphene at $4 \mathrm{~K}$ appears to be $3500 \Omega / \square$.

After the deposition of Co NPs, the sheet resistance of graphene at room temperature is significantly reduced down to $30 \Omega / \square$ (Fig. 4).

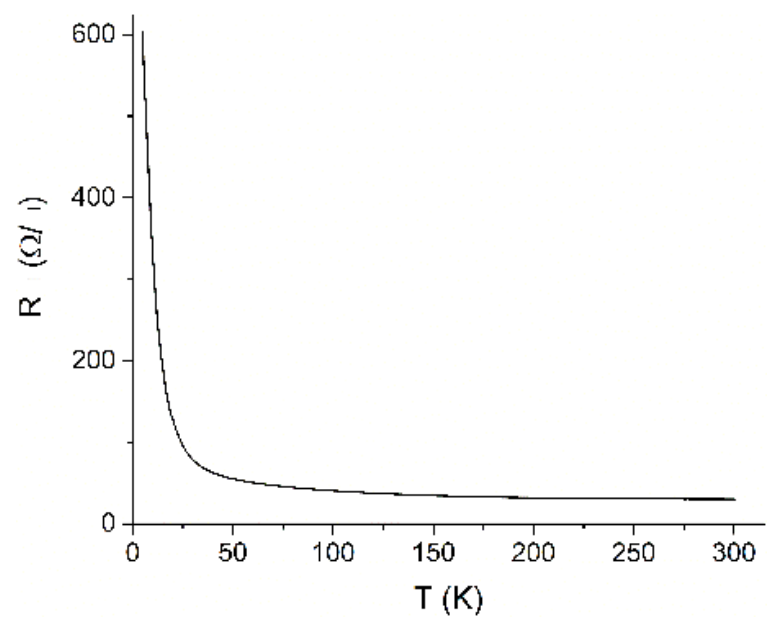

Fig. 4. Temperature dependence of sheet resistance of graphene on $\mathrm{SiO}_{2}$-on-Si substrate after the electrochemical deposition of Co NPs.

The negative value of the resistance temperature coefficient is preserved but the type of the dependence has changed to become more sharp at low temperatures. It could be caused by the changes in the activation energy of the electric conductivity.

We suppose that the observed reduction of the sheet resistance is probably due to the shunting of grain boundaries by Co NPs. In turn, this confirms the formation of ohmic contacts between Co NPs and graphene.

\section{Conclusion}

The proposed modification method of carrier transport in CVD graphene by the Co NPs electrochemical deposition allows to reduce its sheet resistance at room temperature down to $30 \Omega / \square$. Since the Raman spectroscopy study did not reveal any significant changes in the graphene structure after this modification, the observed reduction of sheet resistance can be ascribed to the shunting of disordered grain boundaries by metallic NPs. Moreover, the reduction of optical transparency caused by the electrochemical deposition is only $5 \%$ according to SEM results. All of the above allows to consider the proposed CVD method graphene modification as promising for creating transparent flexible graphene-based films suitable for optoelectronic applications.

\section{Acknowledgments}

The authors from Belarus acknowledge the financial support from the State Committee for Science and 
Technology of the Republic of Belarus (BRFFR project F18PLSHG-005) and the State Program "Photonics, opto- and microelectronics" (project $\mathrm{N}$ 3.3.01). Maxim Rybin thanks the RFBR for support, research project No. 16-32-60203 mol_a_dk.

\section{References}

1. Y. Xu, H. Yu, C. Wang, J. Cao, Y. Chen, Zh. Ma, Y. You, J. Wan, X. Fang and X. Chen, Nanoscale Res. Lett. 12, 254 (2017).

2. D. Kim, D. Lee, Y. Lee and D. Y. Jeon, Adv. Funct. Mater. 23, 5049 (2013).
3. M. Rybin, A. Pereyaslavtsev, T. Vasilieva, V. Myasnikov, I. Sokolov, A. Pavlova, E. Obraztsova, A. Khomich, V. Ralchenko and E. Obraztsova, Carbon 96, 196 (2016).

4. V. G. Bayev et al., Appl. Surf. Sci. 440, 1252 (2018).

5. M. G. Rybin, V. R. Islamova, E. A. Obraztsova and E. D. Obraztsova, Appl. Phys. Lett. 112, 033107 (2018).

6. A. Das, S. Pisana, B. Chakraborty, S. Piscanec, S. K. Saha, U. V. Waghmare, K. S. Novoselov, H. R. Krishnamurthy, A. K. Geim and A. C. Ferrari, Nat. Nanotechnol. 3, 210 (2008). 